\title{
Observations on Sum User Rate for Cellular Downlink
}

\author{
Alex Fridman, Jeffrey Wildman, Steven Weber \\ \{af59,jww33\}@drexel.edu, sweber@ece.drexel.edu \\ Drexel University \\ Philadelphia, PA, USA
}

\begin{abstract}
In this paper, we consider cellular downlink communication from a set of fixed stations to a set of users with uncertain locations modeled by a spatial distribution. Each user is associated with a transmitter through association zones that maximize the signal-to-interference-plus-noise ratio (SINR) to that user. We define an expected spatial capacity metric that captures the expected rate summed over all of these zones. Network performance is measured using an expectation of the spatial capacity, subject to the assumed user density function. We examine the sensitivity of this performance metric to the number of stations and the number of orthogonal (frequency or time) channels, and present some counter-intuitive properties of this metric through illustrative examples, simulation, and optimization.
\end{abstract}

Index Terms-Downlink, cellular networks, capacity, scheduling.

\section{INTRODUCTION}

\section{A. Motivation and Summary}

We consider a cellular downlink scenario where a collection of base stations transmit data to a population of receivers over a collection of orthogonal (time or frequency) channels. Each base station selects at most one channel for transmission. Each receiver associates with the base station (and thereby the corresponding channel) for which the signal to noise plus interference ratio (SINR) is largest, where the downlink interference comes from all co-channel base stations. Each base station, in turn, splits its transmission times fairly among the various receivers with which it is associated. We demonstrate that the expected sum rate under this association rule, assuming a sufficiently large population of users so that all transmitters have at least one associated receiver, is independent of the number of users. Here, the expectation is with respect to the distribution of receiver locations. We call this number the downlink expected spatial capacity since it is the sum, over all base station zones, of the expected received rate over all receiver locations in the zone, normalized by the probability of a receiver being located in the zone. By defining our performance metric as an expectation with respect to receiver locations, our focus is naturally on scenarios where the transmitter channel assignment timescale is long relative to the receiver position timescale, i.e., we compute the expected sum rate (with respect to the assumed receiver location distribution) over all receivers for a fixed transmitter channel assignment.

This metric, although having a natural and intuitive interpretation, is shown in this paper through two 'experiments' to have several counter-intuitive properties. In the first experiment we demonstrate that the spatial capacity is highly sensitive to the transmitter locations, and that 'random' placements have spatial capacities significantly lower than optimal placements. Secondly, we show that adding an additional transmitter can cause a larger increase in spatial capacity when the transmitter is placed near an existing transmitter than when placed so the transmitters are 'evenly spaced'. The results of the second experiment suggest that splitting the transmissions across orthogonal channels (time or frequency) may decrease the spatial capacity, i.e., performance is optimal when all transmitters share a common resource.

Our work is motivated by recent and growing interest in cognitive cellular networks, where downlink transmissions may be optimized by scheduling in time or frequency, by power control, or by various receiver association rules. Indeed, coordinated transmissions and interference mitigation amongst geo-proximate basestations in cellular networks, also known as Coordinated MultiPoint (CoMP) transmission, is a recent inclusion in the 3GPP LTE-Advanced standard [1].

The rest of this paper is organized as follows. $\S I I$ details our network model and defines the spatial capacity. §III describes the results of two simulation experiments. We conclude in $\S \mathrm{IV}$ and provide possible directions for future work.

\section{B. Related Work}

[2] take a game-theoretic approach to determining station placement and frequency band selection in a linear network with uniform power. In a fixed-station approach, they model mobile-users with a uniform density over a line-segment, specify SINR-based user-to-station association rules, and are primarily concerned with network performance in the uplink direction. It is worth emphasizing that our approach is not game-theoretic precisely because of our assumed max SINR association rules. If instead, a receiver associates with a transmitter based on its 'multiplexed' received rate (i.e., Shannon capacity divided by the number of simultaneously supported receivers), then there are potentially competing incentives to associate with both 'strong' transmitters and transmitters with low receiver counts.

The study of fairness and load balancing amongst users and stations has been addressed by several papers [3]-[6]. [3] propose cross-layer, scheduling and load-aware association rules and evaluate their effectiveness via simulation. [4] propose both off- and on-line algorithms to compute handoff and association rules to achieve network-wide proportial fairness 
in user rates. Emphasis is placed on avoiding inter-cell interference at cell boundaries while performing load balancing across stations. [5] explore association rules to promote rate fairness amongst the set of users associated with a common station. A station's signal strength, in addition to its loading (from users' traffic demands), is considered in building such association rules.

[7] examines outage probability and average and minimum per-user rates within multi-tiered wireless cellular systems using a framework rooted in stochastic geometry. They model stations and users as independent Poisson $\mathrm{P}$ oint Processes on an infinite space, whereas we study a finite arena with a fixed but arbitrary number and locations of stations. We model user positions with an arbitrary density function defined over the arena. [7] define per-user rates as the ratio of the expectations of per-cell throughput and number of users per cell, and show that without cell biasing and under certain interference-regimes, their metrics are highly invariant to the number of tiers and density of stations. In contrast, we our metric can be interpreted as the expectation of the ratio (as opposed to the ratio of the expectations), which may reveal interesting behavior in the expected per-user rate, particularly for stations with few users (small association zones).

[8] propose associating users to base stations via a sum rate maximization problem. Of import, their sum rate expression, like ours, accounts for the fact that a station's resources are split amongst its associated users. They develop an upper bound on the maximization problem, while also exploring power-based association rules as a heuristic to the problem. They provide results on their upper bound and heuristics employed under specific instances of station and user placements, whereas we are interested in characterizing the expected sum of rates in the network given uncertain user locations.

[9] propose related metrics which they term spatial spectral efficiency and spatial capacity in the context of ad hoc networks. Transmitter locations follow a homogeneous Poission Point Process (PPP), but each transmitter is limited to a single receiver positioned a fixed distance away from the 'associated' transmitter. Spatial spectral efficiency is defined as an expectation of the sum spectral efficiencies of successful communications, taken over all realizations of the PPP, while spatial capacity is the maximum spatial spectral efficiency such that all communication attempts are successful. Their performance metrics are characterized and bounded analytically as a parameter of the PPP intensity using stochastic geometry techniques.

In our work, we consider SINR-based user association rules that map receiver locations to transmitters, which closely relates to the concept of reception zones, or cells. Reception zones indicate regions where each transmitter can be heard successfully in the presence of the other interfering stations. Several works have focused on the geometric properties formed by reception zones, particularly in the case of uniform power assignment across stations. For example, [10], [11] establish convexity of SINR cells under certain parameter regimes of the SINR model while [10], [12] explore connections between the zones and Voronoi cells. The effect of non-uniform power on the properties of SINR cells has been investigated in [13] while interference cancellation is taken into account in [14]; both works develop refinements to point-location type algorithms. Non-uniform power can break convexity of SINR cells as well as produce a disconnected zone associated with a single transmitter, while interference cancellation can be used to increase the effective area of reception zones.

Finally, SINR cells have also been investigated from the perspective of stochastic geometry, where transmitter positions are not fixed, but set according to a random process. [12] present a general model for wireless communications, including fading effects and heterogeneous power assignments. They investigate properties of SINR cells such as SINR cell volume, SINR cell overlaps, and SINR cell connectivity. They also comment on the transition of SINR cell shapes to disks to Voronoi cells under different model parameter regimes.

\section{Network Model \& Spatial Capacity}

\section{A. Notation \& Model}

We consider a bounded arena $\mathcal{A} \subset \mathbb{R}^{2}$ with $n$ fixed wireless station locations $x_{1}, \ldots, x_{n} \in \mathcal{A}^{n}$. Let $p_{1}, \ldots, p_{n} \in \mathbb{R}^{+}$be the transmit powers, let $\eta \geq 0$ be the background noise power as measured over the system bandwidth, $B>0$, and $l(\cdot, \cdot)$ : $\mathbb{R}^{2} \times \mathbb{R}^{2} \rightarrow \mathbb{R}^{+}$be the pathloss model. Each station is assigned to be active in at most one of $C$ orthogonal channels (e.g. time slots or frequency bands). Let $\mathcal{C}_{c}$ be the set of stations assigned to channel $c$, and let $c(i)$ be the channel assigned to station $i$. The SINR measured at point $y$ from the station at $x_{i}$ is:

$$
\begin{aligned}
\operatorname{sinr}^{f}\left(x_{i}, y\right) & =\frac{p_{i} l\left(x_{i}, y\right)}{\sum_{\hat{i} \in \mathcal{C}_{c(i)} \backslash i} p_{\hat{i}} l\left(x_{\hat{i}}, y\right)+\eta / C} \\
\operatorname{sinr}^{t}\left(x_{i}, y\right) & =\frac{p_{i} l\left(x_{i}, y\right)}{\sum_{\hat{i} \in \mathcal{C}_{c(i)} \backslash i} p_{\hat{i}} l\left(x_{\hat{i}}, y\right)+\eta},
\end{aligned}
$$

where interference is summed over co-channel stations. $\operatorname{sinr}^{f}$ considers the case where channels are represented by evenly spaced frequency bands over $B$, while $\operatorname{sinr}^{t}$ considers the case where channels are represented by evenly spaced time slots over a frame.

Let $\mathcal{H}_{i}, i \in[n]$ be subsets of $\mathcal{A}$, called association zones, where $\mathcal{H}_{i}$ represents the set of locations $y \in \mathcal{A}$ where the association rule, $\phi_{i}(y) \equiv \mathbf{1}\{\cdot\}$, is satisfied:

$$
\mathcal{H}_{i} \equiv\left\{y: \phi_{i}(y)=1, y \in \mathcal{A}\right\}, \quad i \in[n] .
$$

We require that the rules $\phi_{i}, i \in[n]$ form a partition on $\mathcal{A}$, so that every potential user location $y$ associates with a station. In this paper, we create subsets $\mathcal{H}_{i}$ that contain points where station $i$ is perceived to be the strongest station:

$$
\phi_{i}(y)=\mathbf{1}\left\{\operatorname{sinr}\left(x_{i}, y\right)=\max _{\hat{i} \in[n]} \operatorname{sinr}\left(x_{\hat{i}}, y\right)\right\}, \quad i \in[n] .
$$


The metric we consider is based on a user density function $\lambda(y), y \in \mathcal{A}$ such that $\lambda(y) \geq 0$ and $\int_{\mathcal{A}} \lambda(\mathrm{d} y)=1$, representing the spatial distribution on user locations or communication requests with the $n$ stations. Considering a user density function can be useful in situations where exact user positions are not known, e.g. users with mobility within the arena. Let $m$ be a given number of users, and let $Y_{1}, \ldots, Y_{m}$, representing their locations, be iid with density $\lambda$. Let $P_{i}=\int_{\mathcal{H}_{i}} \lambda(\mathrm{d} y)$ be the probability of a user residing in zone $\mathcal{H}_{i}$. Finally, in this paper, we will frequently assume a uniform user density: $\lambda(y)=1 /|\mathcal{A}|$. In this scenario, $P_{i}=\left|\mathcal{H}_{i}\right| /|\mathcal{A}|$.

\section{B. Spatial Capacity}

For our discussion involving $\psi_{\text {cap }}$, let $k_{i}$ be the number of users falling within zone $\mathcal{H}_{i}$. We use $i(y)$ to denote the station whose zone contains point $y$.

Definition 1 (Per-User Capacity): The per-user capacity $\psi_{\text {cap }}(y), y \in \mathcal{A}$ is the capacity of the link between a user located at $y$ and its associated station:

$$
\psi_{\text {cap }}(y)=\frac{B}{C k_{i(y)}} \log _{2}\left(1+\sin r\left(x_{i(y)}, y\right)\right) \quad(\mathrm{bps})
$$

$\psi_{\text {cap }}(y)$ measures the 'time-averaged' rate available to a user at $y . \psi_{\text {cap }}(y)$ takes into account the fact that each station $i$ must multiplex resources amongst its $k_{i}$ associated users. Thus, a user at $y$ gets a $1 / k_{i(y)}$ share of the rate it would have achieved had it been the only user in the zone. Further, the time-averaged per-user rate at $y$ contains a factor of $1 / C$ regardless of whether channels are represented as timeslots or frequency bands. The user at $y$ either gets a constant $B / C$ bandwidth signal from station $i$ or a $B$-bandwidth signal from station $i$ over the duration of one slot per frame $(1 / C$ of the time). What does change in the metric, depending on the choice of channel representation, is the equation for sinr as shown in (1) and (2).

Definition 2 (Spatial Capacity): In a network of $m$ users with locations $\mathbf{y}=\left(y_{1}, \ldots, y_{m}\right)$, the spatial capacity, $\psi_{\text {cap }}^{m}(\mathbf{y})$, is the sum of the per-user capacities divided by the volume of the network arena:

$$
\psi_{\text {cap }}^{m}(\mathbf{y})=\frac{1}{|\mathcal{A}|} \sum_{j \in[m]} \psi_{\text {cap }}\left(y_{j}\right) \quad\left(\mathrm{bps} / \mathrm{m}^{2}\right) .
$$

Proposition 1 (Expected Spatial Capacity): Let $\mathbf{Y}=\left(Y_{1}, \ldots, Y_{m}\right)$ be the random locations of $m$ users, chosen iid according to $\lambda$. The expected spatial capacity is:

$$
\begin{aligned}
\mathbb{E}\left[\psi_{\text {cap }}^{m}(\mathbf{Y})\right]= & \frac{1}{|\mathcal{A}|} \sum_{i \in[n]} \frac{\left(1-\left(1-P_{i}\right)^{m}\right)}{P_{i}} * \\
& \int_{\mathcal{H}_{i}} \frac{B}{C} \log _{2}\left(1+\operatorname{sinr}\left(x_{i(y)}, y\right)\right) \lambda(\mathrm{d} y)
\end{aligned}
$$

Proof: A proof sketch follows. By linearity of expectation, we can express the expected sum capacity as the sum of expected per-user capacities. It then suffices to analyze $\mathbb{E}\left[\psi_{\text {cap }}(Y)\right]$ for an arbitrary user, $Y=Y_{j}$. Condition on $Y \in \mathcal{H}_{i}$, then condition on the random number of users, $K_{i}$, in zone $\mathcal{H}_{i} . \mathbb{P}\left(K_{i}=k_{i} \mid Y \in \mathcal{H}_{i}\right)$ can be modeled by a binomial distribution $\operatorname{Bin}\left(m-1, P_{i}\right)$ due to the fact that $Y$ is already in $\mathcal{H}_{i}$ and the fact that all user locations are iid. The remaining steps are algebraic manipulations of the resulting expression.

We now examine the expected spatial capacity metric (7) under the assumptions of many users and uniform user density.

Proposition 2 (Uniform Density): Given a uniform user density, the expected spatial capacity (7) is:

$$
\begin{aligned}
& \mathbb{E}\left[\psi_{\text {cap }}^{m}(\mathbf{Y})\right]=\frac{1}{|\mathcal{A}|} \sum_{i \in[n]} \frac{\left(1-\left(1-\frac{\left|\mathcal{H}_{i}\right|}{|\mathcal{A}|}\right)^{m}\right)}{\left|\mathcal{H}_{i}\right|} * \\
& \int_{\mathcal{H}_{i}} \frac{B}{C} \log _{2}\left(1+\sin r\left(x_{i(y)}, y\right)\right) \mathrm{d} y \text {. }
\end{aligned}
$$

Proof: Under the assumption of uniform user density, we have $\lambda(y)=\frac{1}{|\mathcal{A}|}$ and $P_{i}=\frac{\left|\mathcal{H}_{i}\right|}{|\mathcal{A}|}$.

Proposition 3 (Many-Users): Given non-empty association zones, the many-users limit $m \rightarrow \infty$ of the expected spatial capacity (7) becomes:

$\mathbb{E}\left[\psi_{\text {cap }}^{m}(\mathbf{Y})\right]=\frac{1}{|\mathcal{A}|} \sum_{i \in[n]} \frac{1}{P_{i}} \int_{\mathcal{H}_{i}} \frac{B}{C} \log _{2}\left(1+\sin r\left(x_{i(y)}, y\right)\right) \lambda(\mathrm{d} y)$.

Proof: Since $P_{i}>0$, we have $\left(1-\left(1-P_{i}\right)^{m}\right) \rightarrow 1$ as $m \rightarrow \infty$.

Proposition 4 (Many-Users, Uniform Density): Given nonempty association zones and a uniform user density, the manyusers limit $m \rightarrow \infty$ of the expected spatial capacity (7) becomes:

$\mathbb{E}\left[\psi_{\text {cap }}(Y)\right]=\frac{1}{|\mathcal{A}|} \sum_{i \in[n]} \frac{1}{\left|\mathcal{H}_{i}\right|} \int_{\mathcal{H}_{i}} \frac{B}{C} \log _{2}\left(1+\operatorname{sinr}\left(x_{i(y)}, y\right)\right) \mathrm{d} y$

Proof: The proof follows from the proofs of Propositions 2 and 3.

Note that the resulting expressions from Propositions 2 through 4 are a sum of integrals of the non-multiplexed capacity equation (5) with $k_{i(y)}=1$. Also note that $\mathbb{E}\left[\psi_{\text {cap }}(Y)\right]$ is independent of the number of users in the network, but still depends on the distribution of users. If we express the expected spatial capacity in terms of the expected per-user capacity directly from (6):

$$
\mathbb{E}\left[\psi_{\text {cap }}^{m}(\mathbf{Y})\right]=\frac{1}{|\mathcal{A}|} \sum_{j \in[m]} \mathbb{E}\left[\psi_{\text {cap }}\left(Y_{j}\right)\right]=\frac{m}{|\mathcal{A}|} \mathbb{E}\left[\psi_{\text {cap }}(Y)\right]
$$

we note that it bears a similarity to the results of Theorem 1 in [9]. Aside from the facts that $i$ ) we are summing per-user 
capacity instead of a per-transmission spectral efficiency in (6) and $i i)$ we include an additional $1 / k_{i(y)}$ multiplexing factor, our spatial capacity can be interpreted as the intensity of users, $\frac{m}{|\mathcal{A}|}$, multiplied by the expected per-user capacity, $\mathbb{E}\left[\psi_{\text {cap }}(Y)\right]$.

Finally, the integral within (7) can be interpreted as the total throughput offered by station $i$ (analagous to $\mathcal{R}_{k}$ in [7]) and the factor $1 / P_{i}$ can be related to the number of users associated with cell $i$ (analagous to $\mathcal{N}_{k}$ in [7]). However, in [7], expectations of both are taken across user and station processes prior to examining the

\section{Simulation AND Optimization ExPERIMENTS}

We illustrate the spatial capacity metric by considering a collection of scenarios with varying node density, node placement, number of schedule slots, and active transmitter sets across multiple slots. The intention of these simulation and optimization experiments is to gain insight into the properties of the metric and how it may be used to improve expected network performance. The metric under consideration is sensitive to all the parameters described in the previous section including the receiver density, noise power, pathloss exponent, arena size, transmit power. We limit our attention to the base case under this model of uniform power allocation, uniform receiver density, isotropic antenna radiation patterns, and noise/transmit power representative of a noise-limited environment.

Unless otherwise stated, we consider a square arena $\mathcal{A}$ of $1 \mathrm{~km}$ by $1 \mathrm{~km}$, transmit power $p_{i}=0.1 \mathrm{~W}$ on every node $i \in[n]$, noise power $\eta=10^{-6} \mathrm{~W}$, and bandwidth $B=10 \mathrm{MHz}$. The pathloss model is assumed to be free space (pathloss exponent of 2). For visualization we use the capacity metric in (5) based on the SINR equation in (2) (with $k_{i}(y)$ set to 1 ) to indicate the maximum capacity available to a receiver placed in a specific location in the arena. For observing the performance of the network as we increase the number of transmitter or scheduling slots, we use the spatial capacity metric in (10).

\section{A. Spatial Density of Transmitters}

One of the central questions to consider in optimizing receiver-agnostic spatial capacity is the effect of transmitter placement and density. The following two experiments suggest, through simulation, the counter-intuitive notion that the optimal position for an additional transmitter is in the vicinity of another active transmitter. So while the mean spatial capacity increases with transmitter density, it is much lower that the spatial capacity achievable through optimization of node placement.

1) Illustrative Transmitter Location Example: In order to introduce the visualization techniques used in this paper and to motivate the transmitter density experiment that follows, we consider two network topologies shown in Fig. 1. The first topology has nodes located $600 \mathrm{~m}$ apart at positions: $(200,200),(200,800),(800,200)$, and $(800,800)$. The second topology has nodes located $200 \mathrm{~m}$ apart at positions: $(400,400),(400,600),(600,400)$, and $(600,600)$. Both networks are located in a $1 \mathrm{~km}$ by $1 \mathrm{~km}$ arena.

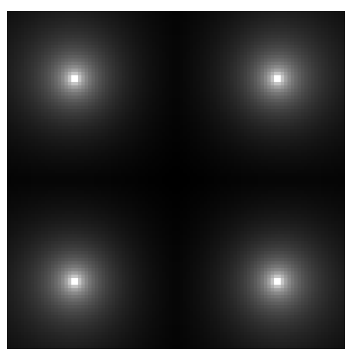

(a) Capacity for topology 1 .

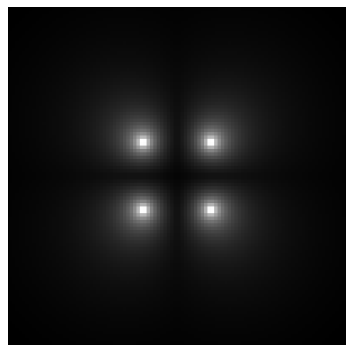

(c) Capacity for topology 2 .

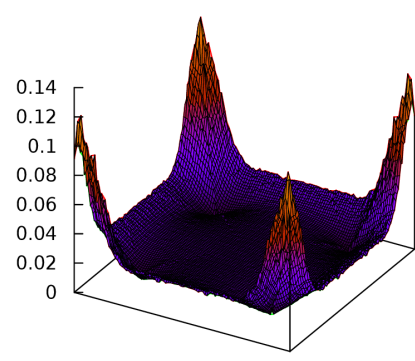

(b) Change for topology 1 .
Fig. 1. On the left is the capacity metric for two topologies of 4 nodes. The gradient for the heatmap is from black to white, or from minimum measured capacity to maximum measured capacity, respectively. On the right is the capacity change metric that quantifies the relative increase in spatial capacity in the event of an additional transmitter added at the sampled location.

The left side of Fig. 1 is a heatmap that shows the capacity metric for these two topologies. On the right side of Fig. 1 is a $3 \mathrm{D}$ visualization of a spatial capacity change metric that measures for a position $y \in \mathcal{A}$ the relative change in spatial capacity (10) in the event that a new transmitter is placed at $y$. A change metric value of zero means that the spatial capacity did not change with the addition of a transmitter. A change metric value of 0.1 means that spatial capacity increased by $10 \%$.

We gather four interesting observations from this experiment. First, placing a transmitter anywhere in the arena will have a non-negative effect on the spatial capacity. Second, generally, the optimal placement for an additional transmitter appears to be close to at least one other transmitter, but not right next to it. In other words, there is a sweet spot in the area near other transmitters where the increase in spatial capacity would be locally optimal were a new transmitter positioned there. This is a counter-intuitive observation that suggest a highly-clustered topology is likely to lead to a higher spatial capacity than a less-clustered one. Third, topology 1 indicates that edge effects may have a significant influence on the metric. Transmitters close to the edge of the arena have a cell that is smaller than it otherwise might be. The counterintuitive observation here is that such a curtailed cell often leads to an increase in spatial capacity.

The fourth observation is that the change metric for the majority of the arena is close to zero, increasing significantly only in the aforementioned zones near other transmitters. Therefore, if we were to randomly place a transmitter in the arena, the expected improvement will be much lower than the 


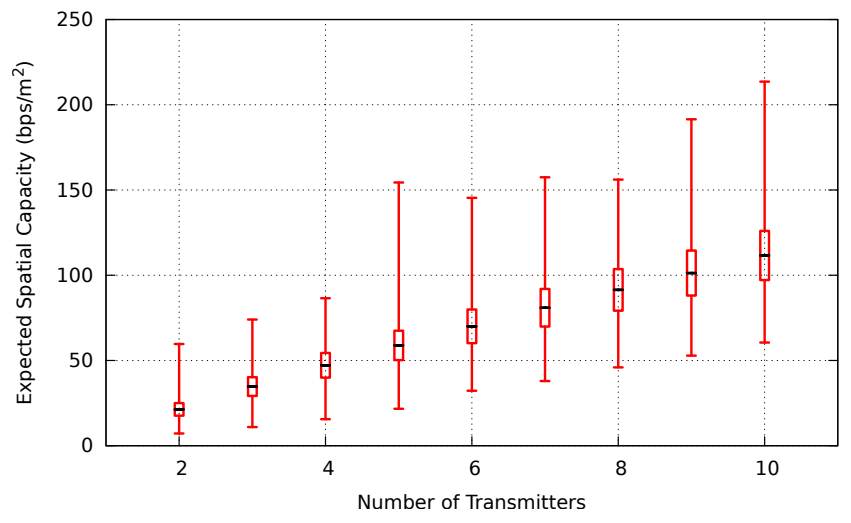

Fig. 2. Box plot showing the spatial capacity for 10,000 realizations of $n \in\{2,3, \ldots, 10\}$. The middle line is the mean. The box is the mean plus/minus the standard deviation. The whiskers of the plot are the minimum and maximum of each set of 10,000 spatial capacity values.

maximum improvement if we optimize the placement of the transmitter. This observation is built upon in the next section.

2) Varying Number of Transmitters: In this experiment, we consider the relationship between the spatial capacity and the number of active transmitters in the arena. For each $n \in\{2,3, \ldots, 10\}$ number of transmitters, we simulate 10,000 realizations of random node positions, and compute the spatial capacity of the network assuming all $n$ transmitters are actively transmitting in a single slot. Fig. 2 shows the mean, standard deviation, and range of the 10,000 spatial capacity evaluations for each $n$. The node topologies are generated using a 2D uniform Binomial Point Process (BPP).

We can draw two insights from the data in Fig. 2. First, the mean spatial capacity increases with the number of transmitters. Second observation is that the mean spatial capacity is much closer to the minimum than the maximum. This is related to the observation in $\S$ III-A1 that the majority of two-node configuration in the arena do not lead to a spatial capacity value that is much higher than if just one of these nodes was transmitting. While this cannot be directly extended to arbitrary configurations of $n$ nodes, it does provide some intuition that clustered topologies (with small groups of nodes positioned close together) provide higher spatial capacity than topologies that are more uniformly spread out in the arena.

\section{B. Scheduling}

The optimal schedule problem seeks a set of channel assignments $c(i), i \in[n]$ in order to maximize some expected performance metric $\mathbb{E}[\psi(Y)]$ of the system. In the following two experiments, we consider multiplexed association zones (see $\S$ II-A) and optimal packing of transmissions over multiple schedule slots.

1) Illustrative Multiplexing Example: We consider two examples of 2-slot assignments for network of 25 nodes positioned in a lattice of $5 \times 5$. Fig. 3 shows the visualization of per-slot capacity and the multiplexed capacity for both of these assignments. Moreover, it shows the association zones for a multiple-slot case based on which the spatial capacity

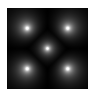

(a) 1 slot

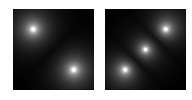

(b) 2 slots.

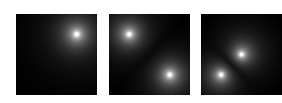

(c) 3 slots. (d) 4 slots.

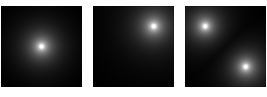

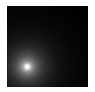

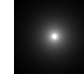

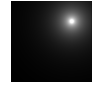

(e) 5 slots
Fig. 4. The optimal slot assignments for a network of five nodes when the number of slots is constrained to $1,2,3,4$, or 5 .

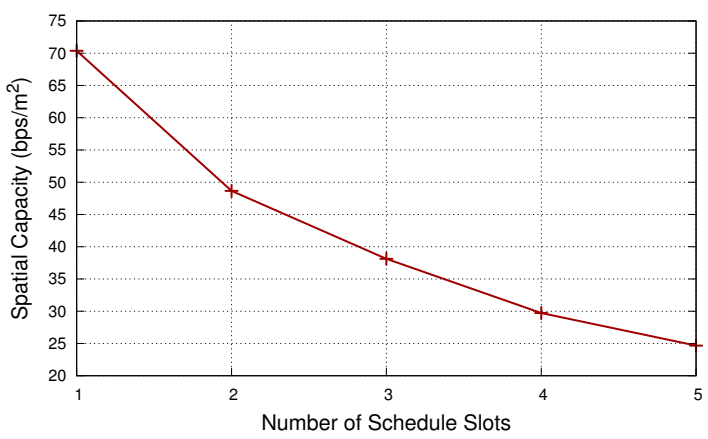

Fig. 5. The spatial capacity of multiplexed association zones from optimal scheduling on 1, 2, 3, 4, and 5 schedule slots as visualized in Fig. 4.

metric is computed. An association zone $\mathcal{H}_{i}$ in (3) for node $i \in[n]$ is a subset of the arena in which the capacity metric is maximized for a receiver that can optimally choose in which slot to listen in on. In the homogeneous power single slot case, the association zones form a Voronoi diagram of convex cells [10]. In the multiple-slot case, as shown in Fig. 3, the zones can be convex, but can also be disjoint non-convex with holes.

These two examples of slot assignments were chosen to demonstrate the counter-intuitive nature of the spatial capacity metric in its valuation of the density of transmitters over the negative interference effects on the capacity of each individual cell. It may seem reasonable to assume that the more even split of slot assignments in the first example would lead to an orderof-magnitude higher spatial capacity. Instead the difference is small with spatial capacity of $259.4 \mathrm{bps} / \mathrm{m}^{2}$ for the first example and $186.4 \mathrm{bps} / \mathrm{m}^{2}$ for the second example.

2) Varying Number of Schedule Slots: In this experiment, we compute an optimal schedule for a network of five nodes in a topology shown in Fig. 4(a). We find the schedule for 1, 2, 3, 4 , and 5 slot assignments for which the multiplexed association zones maximize the spatial capacity. Fig. 4 shows the optimal solution for each of these five optimization problems. Each transmitter is constrained to only transmit in a single time slot. Fig. 5 shows the decreasing spatial capacity for each of these solutions as the number of slots increases.

The fact that the spatial capacity decreases with the number of schedule slots confirms the counter-intuitive notion (from $\S$ III-A) in this specific case that the spatial capacity is maximized when all transmitters are active. 

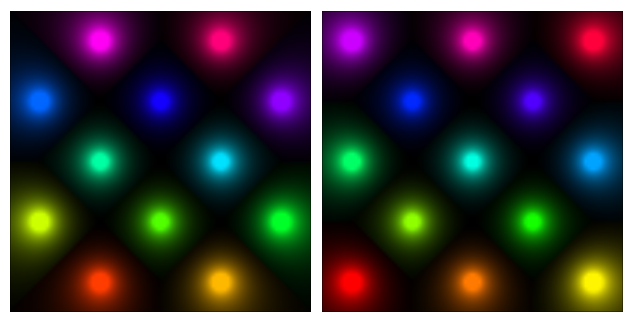

(a) Ex. 1: Capacity of nodes active in slots 1 and 2.
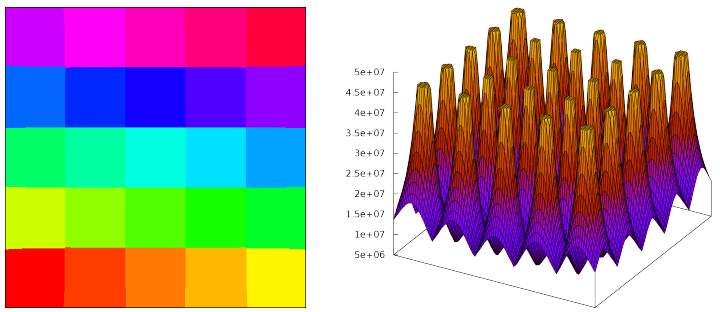

(c) Ex. 1: Multiplexed asso- (d) Ex. 1: Capacity of multiplexed ciation zones (see $\S I I-A)$. $\quad$ schedule.

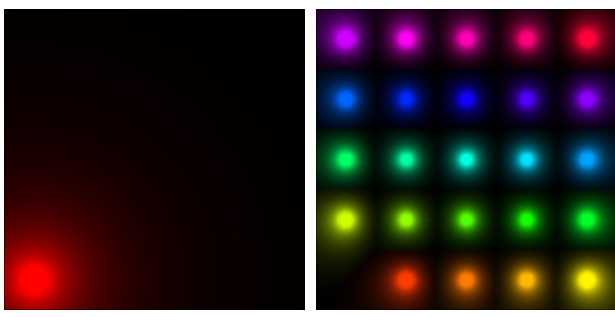

(b) Ex. 2: Capacity of nodes active in slots 1 and 2.
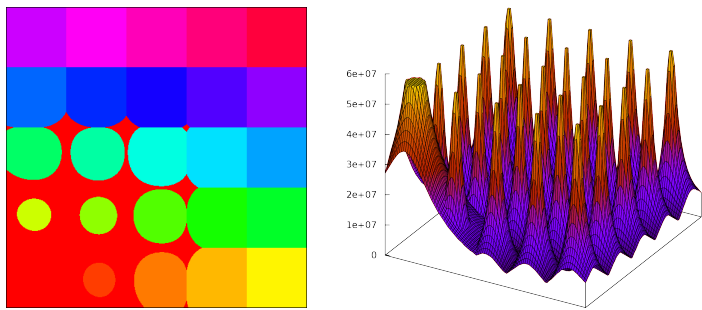

(e) Ex. 2: Multiplexed asso- (f) Ex. 2: Capacity of multiplexed ciation zones (see $\S$ II-A). $\quad$ schedule.

Fig. 3. Two illustrative examples of channel assignments on a network of 25 nodes that are positioned in a $5 \times 5$ lattice. The first row shows the transmitters assigned to the first and second slots for both examples. The second row shows the multiplexed association zones and multiplexed capacity metric (in bps) for both examples. The spatial capacity is $259.4 \mathrm{bps} / \mathrm{m}^{2}$ for the first example and $186.4 \mathrm{bps} / \mathrm{m}^{2}$ for the second example.

\section{CONCLusions \& Future Work}

In this paper, we presented a spatial capacity metric computed over SINR-based association regions in the context of cellular downlink communication. We considered the sensitivity of this metric to the position, density, and scheduling of transmitters to reveal some counter-intuitive properties of this metric. In particular, we observed: (1) that a highlyclustered topology leads to a higher spatial capacity than a less-clustered one, (2) that spatial capacity increases as the number of transmitters increases, and decreases as the number of schedule slots increases.

Future work will expand the network model on which the spatial capacity metric is based to include heterogeneous power and directional antennas, and expand the optimization framework to include power control and facility location problems. In $\S$ III-A1, we observed that adding a transmitter anywhere in the arena has a non-negative effect on spatial capacity. This observation was gathered from extensive simulation. In future work, we seek to prove this property for arbitrary node placement configurations.

\section{ACKNOWLEDGMENTS}

This work is partially funded by the National Science Foundation (CNS-1147838).

\section{REFERENCES}

[1] M. Sawahashi, Y. Kishiyama, A. Morimoto, D. Nishikawa, and M. Tanno, "Coordinated multipoint transmission/reception techniques for lte-advanced [coordinated and distributed mimo]," IEEE Wireless Communications Magazine, vol. 17, no. 3, pp. 26-34, June 2010.

[2] E. Altman, A. Kumar, C. Singh, and R. Sundaresan, "Spatial sinr games of base station placement and mobile association," IEEE/ACM Transactions on Networking, vol. 20, no. 6, pp. 1856-1869, December 2012.
[3] A. Sang, X. Wang, and M. Madihian, "Coordinated load balancing, handoff/cell-site selection, and scheduling in multi-cell packet data systems," Wireless Networks, vol. 14, pp. 103-120, January 2008.

[4] K. Son, S. Chong, and G. de Veciana, "Dynamic association for load balancing and interference avoidance in multi-cell networks," IEEE Transactions on Wireless Communications, vol. 8, no. 7, July 2009.

[5] Y. Bejerano, S.-J. Han, and L. E. Li, "Fairness and load balancing in wireless LANs using association control," IEEE/ACM Transactions on Networking, vol. 15, no. 3, pp. 560-573, June 2007.

[6] H. Kim, G. de Veciana, X. Yang, and V. Muthaiah, "Distributed $\alpha$ optimal user association and cell load balancing in wireless networks," IEEE/ACM Transactions on Networking, vol. 20, no. 1, pp. 177-190, February 2012.

[7] H.-S. Jo, Y. J. Sang, P. Xia, and J. G. Andrews, "Heterogeneous cellular networks with flexible cell association: A comprehensive downlink sinr analysis," IEEE Transactions on Wireless Communications, vol. 11, no. 10, pp. 3484-3495, October 2012.

[8] S. Corroy, L. Falconetti, and R. Mathar, "Dynamic cell association for downlink sum rate maximization in multi-cell heterogeneous networks," in IEEE International Conference on Communications (ICC), June 2012, pp. 2457-2461.

[9] P. Nardelli, P. Cardieri, and M. Latva-aho, "Spatial capacity of ad hoc wireless networks with poisson distributed nodes," in IEEE Wireless Communications and Networking Conference (WCNC), April 2012, pp. 878-882.

[10] C. Avin, Y. Emek, E. Kantor, Z. Lotker, D. Peleg, and L. Roditty, "Sinr diagrams: Towards algorithmically usable sinr models of wireless networks," in Proceedings of the 28th ACM Symposium on Principles of Distributed Computing, ser. PODC '09. New York, NY, USA: ACM, 2009, pp. 200-209.

[11] Z. Lotker and D. Peleg, "Structure and algorithms in the sinr wireless model," SIGACT News, vol. 41, no. 2, pp. 74-84, June 2010.

[12] F. Baccelli and B. Baszczyszyn, "Stochastic geometry and wireless networks volume 1: Theory," Foundations and Trends in Networking, vol. 3, no. 3-4, pp. 249-449, 2009.

[13] E. Kantor, Z. Lotker, M. Parter, and D. Peleg, "The topology of wireless communication," in Proceedings of the 43rd Annual ACM Symposium on Theory of Computing, ser. STOC '11. New York, NY, USA: ACM, 2011, pp. 383-392.

[14] C. Avin, A. Cohen, Y. Haddad, E. Kantor, Z. Lotker, M. Parter, and D. Peleg, "Sinr diagram with interference cancellation," in Proceedings of the Twenty-Third Annual ACM-SIAM Symposium on Discrete Algorithms, ser. SODA '12. SIAM, 2012, pp. 502-515. 